\title{
Bienestar psicológico y estrategias de afrontamiento frente a la COVID-19 en universitarios
}

\author{
Psychological well-being and coping strategies in dealing with COVID-19 in university students \\ Jonnathan Harvey Narváez ${ }^{1 *}$ orcid.org/0000-0002-3023-5156 \\ Lina María Obando-Guerrero² orcid.org/0000-0001-6286-5251 \\ Karen Melisa Hernández-Ordoñez' orcid.org/0000-0003-4763-2294 \\ Eliana Katherine De la Cruz-Gordon'1 orcid.org/0000-0001-8700-8109
}

1. Departamento de Psicología, Universidad de Nariño. Pasto, Colombia.

2. Facultad de Psicología, Universidad de Buenos Aires. Buenos Aires, Argentina.

Fecha de recepción: Enero 17 - 2021

Fecha de revisión: Mayo 24 - 2021

Fecha de aceptación: Agosto 30 - 2021

Narváez JH, Obando Guerrero LM, Hernández Ordoñez KM, De la Cruz Gordon EK. Bienestar psicológico y estrategias de afrontamiento frente a la COVID-19 en universitarios. Univ. Salud. 2021;23(3):207-216. DOI: https://doi.org/10.22267/rus.212303.234

\section{Resumen}

Introducción: La pandemia causada por la COVID-19 es una situación sin precedentes a nivel mundial, y es previsible que tenga un importante impacto en los seres humanos, al causar un cambio en las dinámicas, hábitos y rutinas que inciden en el bienestar psicológico y las estrategias de afrontamiento. Objetivo: Identificar si existe asociación entre el bienestar psicológico y las diferentes estrategias de afrontamiento frente al impacto psicosocial de la COVID-19 en universitarios. Materiales y métodos: Estudio cuantitativo, no experimental, de tipo descriptivo-correlacional y transversal, con una muestra de 201 estudiantes universitarios del sur-occidente de Colombia. Se administró la Escala de Bienestar Psicológico de Ryff y la Escala de Estrategias de Coping - Modificada (EEC-M). El análisis de datos se realizó con el Software SPSS. Resultados: Se encontró una asociación directa estadísticamente significativa entre el bienestar psicológico y las estrategias de afrontamiento, solución de problemas, búsqueda de apoyo social, religión, evitación cognitiva, reevaluación positiva y negación. Conclusiones: Las estrategias de afrontamiento se encuentran asociadas con el bienestar psicológico de los estudiantes, y su intervención podría generar insumos importantes para el afrontamiento de las consecuencias psicosociales generadas por la pandemia.

Palabras clave: COVID-19; pandemia; bienestar psicológico; estrategia de afrontamiento. (Fuente: DeCS, Bireme).

\begin{abstract}
Introduction: The pandemic caused by COVID-19 is an unprecedented worldwide situation. It is predicted that COVID-19 will have a significant impact on humans as it can cause changes in their dynamics, habits, and routines that affect their psychological wellbeing and coping strategies. Objective: To identify whether there is an association between psychological well-being and the different coping strategies in confronting the psychological impact of COVID-19 in university students. Materials and methods: A quantitative, non-experimental, descriptive-correlational, and cross-sectional study was carried out on a sample of 201 university students from the southwestern region of Colombia. The Ryff 's Psychological Well-Being Scale and the Modified Coping Strategies Scale (EEC-M) were used. Data analysis was performed with SPSS software. Results: A statistically significant direct association was found between psychological well-being and coping strategies, problem solving, seeking social support, religion, cognitive avoidance, positive reassessment, and denial. Conclusion: Coping strategies are related to the psychological well-being of students, so their use can provide important inputs to face the psychological consequences generated by the pandemic.
\end{abstract}

Keywords: Covid-19; pandemic; psychological well-being; coping strategies. (Source: DeCS, Bireme).

\footnotetext{
*Autor de correspondencia

Jonnathan Harvey Narváez

e-mail: jonnathanharnarvaez@gmail.com
} 


\section{Introducción}

La pandemia por coronavirus representa un gran desafío para la salud mental, debido al enorme impacto psicológico de las medidas de emergencia sanitaria que han tenido lugar en diversos países. La rápida difusión de la enfermedad ha generado imprevistos e importantes cambios en la sociedad a nivel mundial, que van desde sus efectos sanitarios directos en la población, hasta el desplome de la producción, los intercambios comerciales, además de las consecuencias de la emergencia sociosanitaria en la salud mental y la calidad de vida de las personas(1).

En Colombia el 24 de marzo se inició una cuarentena a nivel nacional, decretando el confinamiento obligatorio en toda la población. Es evidente que el impacto causado por el virus se presenta de distinta forma en los pobladores, dadas las condiciones personales y sociales que pueden contribuir a una afectación mayor o menor (2). Lo cierto, es que pese a las diversas políticas y prácticas de autocuidado orientadas a mitigar y controlar la pandemia, esta ha causado problemas financieros, psicológicos $\mathrm{y}$ emocionales en las personas y comunidades(3).

En el plano de la salud mental, debido a lo reciente de la enfermedad, no se cuenta con suficiente evidencia disponible, sin embargo, especialistas de los diferentes ámbitos de la ciencia reportan una serie de reacciones y estados psicológicos observados en la población(4). En los primeros estudios, se sostiene que una de las respuestas emocionales más frecuente ha sido el miedo, incertidumbre, percepciones distorsionadas de riesgo y ansiedad(5). Un efecto similar lo reportan, quienes estudian los mensajes enviados de Weibo (sitio web chino de redes sociales) antes y después de la declaración de la COVID-19 el 20 de enero del 2020, encontrando que las emociones negativas como la ansiedad, la depresión y la indignación, así como la sensibilidad a los riesgos sociales aumentaron, en tanto disminuyeron las emociones positivas como la felicidad y la satisfacción con la vida(6).

Dentro de las medidas para contener el virus, en el sector educativo, los lineamientos del Ministerio de Educación de Colombia llamaron al modelo de aprendizaje virtual desde el trabajo en casa, medida que tomó de improvisto a estudiantes y maestros, y que progresivamente trajo dificultades para la adaptación y representó un factor de riesgo a la salud mental(7). El conjunto de afectaciones derivadas por el paso de la presencialidad a la virtualidad, colocó a prueba los diversos recursos psicológicos, tanto cognitivos, conductuales, afectivos y motivacionales(8), lo que obligó a los estudiantes, a activar estrategias de afrontamiento necesarias para la regulación de estresores y el aprendizaje exitoso(9).

En la educación superior, fue necesario reinventar la forma de enseñar a través del aprovechamiento de los recursos digitales disponibles, sin embargo, la dispersión territorial de los estudiantes, la ineficiente conectividad y carencia de recursos tecnológicos, fueron obstáculos para garantizar el acceso y permanencia en el proceso educativo(10). A nivel tecnológico, no todos los profesores y estudiantes contaban con equipos y habilidades para la gestión del aprendizaje en línea, y la formación profesional no se centra únicamente en la transferencia de

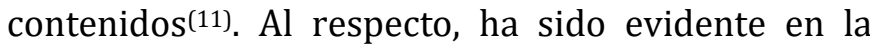
pandemia, que el desarrollo de los individuos no se da en forma aislada del contexto de socialización, las personas y comunidades viven y se relacionan en una compleja red de sistemas sociales, que proveen mediante la interacción, una serie de factores protectores que logran minimizar el impacto psicosocial del riesgo y garantizan fuentes de apoyo(12).

En efecto, el bienestar psicológico es una percepción personal que se va construyendo a través del conjunto de vivencias positivas o negativas del individuo, lo que incluye la calidad en las relaciones interpersonales y la disponibilidad de fuentes de apoyo $^{(13)}$. En este escenario, el afrontamiento es un proceso complejo y multidimensional que tiene gran relevancia en la actualidad ya que se define como los esfuerzos cognitivos y conductuales que se reflejan mediante conducta manifiesta o interna, en el manejo/control de demandas externas (ambiente) como internas (características de la personalidad, estado emocional), y que son evaluadas como algo que excede los recursos o posibilidades de la persona para adaptarse a una situación(14). En sí, las estrategias de afrontamiento constituyen herramientas o recursos que el sujeto desarrolla para hacer frente a demandas específicas, estos recursos pueden ser positivos al permitirles afrontar las demandas de forma adecuada, o negativos, al perjudicar su bienestar biopsicosocial(15).

De ahí la necesidad de proponer un estudio que permita analizar las posibles consecuencias de la exposición general a la pandemia de la COVID-19 
sobre las diversas dimensiones de la salud mental desde una perspectiva biopsicosocial y situada(16). Por lo anterior, el presente artículo busca identificar si existe asociación entre el bienestar psicológico y las diferentes estrategias de afrontamiento frente al impacto psicosocial de la COVID-19 en universitarios.

\section{Materiales y métodos}

El presente es un estudio cuantitativo, no experimental, dado que no se manipuló las variables y se evaluó los constructos tal como se presentan en el contexto universitario del suroccidente colombiano, durante el período de la pandemia a finales de 2020. El tipo de estudio es descriptivocorrelacional y transversal, en el que se busca caracterizar y asociar las variables bienestar psicológico y estrategias de afrontamiento(17).

Se empleó una muestra de tipo no probabilístico e intencional de 201 estudiantes universitarios quienes cumplieron con los siguientes criterios de inclusión: a) personas mayores de 18 años, b) que actualmente se encuentren estudiando en alguna universidad ya sea pública o privada del suroccidente de Colombia (Valle del Cauca, Cauca, Nariño y Putumayo) y c) que aceptaran participar de manera voluntaria en el estudio. Se consideró como criterio de exclusión la no firma del consentimiento informado.

\section{Instrumentos}

Para la recolección de datos se empleó dos instrumentos validados que permiten medir el bienestar psicológico y las estrategias de afrontamiento. Para medir la variable bienestar psicológico se utilizó la versión validada en Colombia de la Escala de Bienestar Psicológico de Ryff(18), la cual es tipo Likert y está constituida por 29 ítems. Tras el análisis factorial confirmatorio se comprobó un modelo teórico de seis factores que cumplió con índices adecuados. La consistencia interna fue medida mediante el alfa omega de McDonald el cual osciló entre 0,60 y 0,83 para las seis escalas.

Para la variable estrategias de afrontamiento se aplicó la EEC-M la cual fue adaptada y validada en Colombia(19). Esta escala de tipo Likert quedó constituida por 69 ítems, que se distribuyeron tras el análisis factorial exploratorio en 12 factores: solución de problemas, apoyo social, espera, religión, evitación emocional, apoyo profesional, reacción agresiva, evitación cognitiva, reevaluación positiva, expresión de la dificultad de afrontamiento, negación y autonomía. La consistencia interna medida por el Alfa de Cronbach fue de 0,84 y la varianza total explicada de $58 \%$.

\section{Procedimiento}

Dadas las condiciones de la pandemia y las medidas de distanciamiento preventivo, una vez culminado el diseño del estudio y la preparación de los instrumentos, se contactó por medios electrónicos un grupo de estudiantes de universidades públicas y privadas de Valle del Cauca, Cauca, Nariño y Putumayo, a quienes se les socializó el objetivo, naturaleza y riesgos del estudio, de modo que con la firma del consentimiento informado aceptaron de manera voluntaria participar de la investigación. Con el permiso otorgado, se procedió a aplicar la batería de instrumentos por medio virtual acorde a las medidas de autocuidado dadas por la contingencia de la COVID-19. Con los resultados obtenidos se realizó un análisis de los datos, los cuales fueron contrastados con otros estudios para la consolidación del informe final.

\section{Análisis de datos}

Para el análisis descriptivo de las variables bienestar psicológico y estrategias de afrontamiento se calculó la media, la desviación estándar, el puntaje mínimo y máximo mediante el software IBM SPSS Stactistics Standard versión 25.0. Posteriormente, para evaluar la distribución de los datos se empleó la prueba de Kolmogorov-Smirnov la cual se aplica para variables cuantitativas continuas, cuando el tamaño muestral es mayor de $50^{(20)}$. De este modo, para analizar la correlación entre las variables y teniendo en cuenta que en el estudio los datos no se distribuyeron normalmente, se aplicó el coeficiente de correlación de Spearman el cual es el análogo no paramétrico de la correlación de Pearson(21).

\section{Consideraciones éticas}

Para garantizar los derechos y el bienestar de los participantes se consideraron los elementos éticos descritos en la Ley 1090 del 2006(22) que obedece al Código Deontológico del Psicólogo y las directrices del Comité de Ética en Investigaciones de la Universidad de Nariño para efectos de investigación con seres humanos, con acta de aprobación 030 de 30 octubre de 2020. En consideración, los participantes de la investigación fueron informados sobre los objetivos, procedimientos y riesgos, de manera que quienes decidieron participar de manera voluntaria firmaron el consentimiento informado $y$ se vincularon al estudio. 


\section{Resultados}

En primera instancia se indagaron algunas variables sociodemográficas y académicas que podrían posiblemente interferir en las asociaciones, se tuvo en cuenta la homogeneidad en la población respecto al género, pertenencia a instituciones públicas $\mathrm{y}$ privadas y al semestre que cursaban los estudiantes (Tabla 1).

Tabla 1. Frecuencias de las variables sociodemográficas $(n=201)$

\begin{tabular}{lrc}
\hline \multicolumn{1}{c}{ Variable } & Frecuencia & $\mathbf{\%}$ \\
\hline Género & & \\
Femenino & 107 & 53,23 \\
Masculino & 94 & 46,77 \\
Institución educativa & & \\
$\begin{array}{l}\text { Privada } \\
\text { Pública }\end{array}$ & 100 & 49,75 \\
Semestre & 101 & 50,25 \\
Primero a quinto & & \\
Sexto a décimo & 101 & 50,25 \\
\hline
\end{tabular}

Con relación a los puntajes obtenidos en la escala de bienestar psicológico, se encontró que los participantes obtuvieron una media de 121,38 y desviación estándar de $\pm 11,16$, el puntaje mínimo fue de 82 y el máximo de 157, por ello se propone que los sujetos presentaron una tendencia de puntajes medios-altos en esta categoría.

Adicionalmente, se realizó un análisis de las dimensiones que componen al bienestar psicológico encontrando la prevalencia de la categoría propósito en la vida $(4,7 \%)$ y autoconcepto $(4,64 \%)$. Con menor frecuencia se encuentra la autonomía $(3,66 \%)$ y las relaciones positivas $(3,88 \%)$.

En lo que respecta a las estrategias de afrontamiento se identificó que las más empleadas por los estudiantes fueron solución de problemas $(3,89 \%)$, reevaluación positiva $(3,75 \%)$, evitación emocional $(3,46 \%)$ y evitación cognitiva $(3,33 \%)$. En menor medida los sujetos emplearon estrategias de afrontamiento como la búsqueda de apoyo profesional $(2,32 \%)$ y espera $(2,61 \%)$.

Por otra parte, en el estudio se buscó determinar la relación entre el bienestar psicológico y las estrategias de afrontamiento para lo cual se aplicó inicialmente la prueba de normalidad KolmogorovSmirnov. Se encontró un valor $p=0,02$ que evidencia que los datos no se distribuyen de forma normal, por ello se aplicaron pruebas no paramétricas; así se utilizó el coeficiente de correlación de Spearman. En la Tabla 2 se muestran las correlaciones encontradas.

Tabla 2. Correlación entre bienestar psicológico y estrategias de afrontamiento

\begin{tabular}{|c|c|c|c|c|c|c|c|c|c|c|c|c|}
\hline & SP & BAS & E & $\mathbf{R}$ & EE & BAP & RA & EC & RP & EDA & $\mathbf{N}$ & A \\
\hline BP & $0,315^{\text {** }}$ & $0,254^{* *}$ & 0,073 & $0,145^{*}$ & 0,084 & 0,074 & $-0,009$ & $0,255^{* *}$ & $0,363^{* *}$ & $-0,025$ & $0,166^{*}$ & 0,003 \\
\hline
\end{tabular}

Nota: $\mathrm{BP}=$ Bienestar psicológico, $\mathrm{SP}=$ Solución de Problemas, $\mathrm{BAS}=$ Búsqueda de Apoyo Social, E= Espera, R= Religión, EE= Evitación Emocional, BAP= Búsqueda de Apoyo Profesional, RA= Reacción Agresiva, EC= Evitación Cognitiva, RP= Reevaluación Positiva, EDA= Expresión de la Dificultad de Afrontamiento, N= Negación, A= Autonomía

A partir de lo expuesto en la Tabla 2, se plantea que existe una correlación directa estadísticamente significativa entre el bienestar psicológico y las estrategias de afrontamiento tales como solución de problemas, búsqueda de apoyo social, religión, evitación cognitiva, reevaluación positiva y negación.

Junto a lo anterior, se correlacionaron las dimensiones de bienestar psicológico con las estrategias de afrontamiento utilizadas por los participantes en el contexto de la pandemia (Tabla 3). En relación al autoconcepto se encontró que tiene una correlación directa estadísticamente significativa con las estrategias de afrontamiento solución de problemas, búsqueda de apoyo social, búsqueda de apoyo profesional, evitación cognitiva y reevaluación positiva. Por el contrario, tiene una relación inversa estadísticamente significativa con espera, evitación emocional, reacción agresiva y expresión de la dificultad de afrontamiento.

En cuanto a la dimensión relaciones positivas, se determinó que está se asocia de manera directa con las estrategias de afrontamiento espera, evitación emocional, expresión de la dificultad de afrontamiento y negación, tomando una postura pasiva frente a lo que está pasando en la vida de los sujetos. En cuanto a la autonomía, se encontraron relaciones directas estadísticamente significativas con las estrategias de afrontamiento espera, evitación emocional, reacción agresiva y expresión de la dificultad de afrontamiento. 
Tabla 3. Correlación entre dimensiones de bienestar psicológico y estrategias de afrontamiento

\begin{tabular}{lrrrrrrrrrrrr}
\hline & SP & \multicolumn{1}{c}{ BAS } & \multicolumn{1}{c}{ E } & \multicolumn{1}{c}{ R } & EE & \multicolumn{1}{c}{ BAP } & \multicolumn{1}{c}{ RA } & EC & RP & EDA & N & A \\
\hline A & $0,352^{* *}$ & $0,347^{* *}$ & $-0,150^{*}$ & 0,086 & $-0,272^{* *}$ & $0,147^{*}$ & $-0,182^{* *}$ & $0,179^{*}$ & $0,382^{* *}$ & $-0,331^{* *}$ & 0,044 & $-0,041$ \\
RP & $-0,100$ & $-0,113$ & $0,177^{*}$ & $-0,028$ & $0,326^{* *}$ & $-0,058$ & 0,100 & 0,020 & $-0,046$ & $0,304^{* *}$ & $0,140^{*}$ & 0,058 \\
Aut & $-0,047$ & $-0,044$ & $0,268^{* *}$ & 0,064 & $0,293^{* *}$ & $-0,064$ & $0,198^{* *}$ & 0,080 & $-0,042$ & $0,328^{* *}$ & 0,064 & 0,065 \\
DE & $0,166^{*}$ & $0,227^{* *}$ & 0,061 & 0,085 & 0,113 & 0,093 & 0,096 & $0,208^{* *}$ & 0,114 & 0,120 & 0,061 & 0,070 \\
PV & $0,437^{* *}$ & $0,239^{* *}$ &,$- 146^{*}$ & $0,195^{* *}$ & $-0,172^{*}$ & $0,146^{*}$ & $-0,156^{*}$ & $0,166^{*}$ & $0,463^{* *}$ & $-0,327^{* *}$ & 0,096 & 0,004 \\
CP & $0,257^{* *}$ & 0,123 & 0,032 & 0,090 & 0,042 & 0,059 & 0,033 & $0,201^{* *}$ & $0,328^{* *}$ & $-0,113$ & $0,169^{*}$ & 0,015 \\
\hline
\end{tabular}

Nota: A=Autoconcepto, RP=Relaciones Positivas, Aut= Autonomía, DE= Dominio del Entorno PV= Propósito de la Vida, $\mathrm{CP}=\mathrm{Crecimiento}$ Personal, $\mathrm{SP}=$ Solución de Problemas, BAS= Búsqueda de Apoyo Social, E= Espera, R= Religión, EE= Evitación Emocional, BAP= Búsqueda de Apoyo Profesional, RA= Reacción Agresiva, EC= Evitación Cognitiva, RP= Reevaluación Positiva, EDA= Expresión de la Dificultad de Afrontamiento, N= Negación, A= Autonomía

Por su parte, la dimensión dominio del entorno tuvo una relación directa con las estrategias solución de problemas, búsqueda de apoyo social y evitación cognitiva, identificando estrategias que ayudaron a los usuarios a sobrellevar e intentar controlar lo que pasa en su entorno en medio de la pandemia. En cuanto a propósitos de la vida se asoció de forma directa con solución de problemas, búsqueda de apoyo social, religión, búsqueda de apoyo profesional, evitación cognitiva y reevaluación positiva. Contrariamente, tiene una relación inversa con las estrategias de espera, evitación emocional y expresión en la dificultad de afrontamiento, por ende, el sujeto prioriza sus objetivos o metas y toman acciones para alcanzarlas. Finalmente, la dimensión crecimiento personal se asocia de manera directa con las estrategias de afrontamiento solución de problemas, evitación cognitiva, reevaluación positiva y negación, lo cual contribuye a un mejor manejo de las circunstancias causadas por la COVID-19.

\section{Discusión}

En relación a la categoría bienestar psicológico, se identificó que los participantes tenían una tendencia a puntajes medios-altos, lo cual indica una correlación directa entre las variables evaluadas, así, las personas que están mejor preparadas para afrontar y resolver situaciones y desafíos en la vida suelen experimentar mayor bienestar psicológico, tienden a ser positivos, cuentan con habilidades para la regulación de emociones, tienen mayores niveles de autoestima alta; lo cual les brinda condiciones de equipamiento psicológico para percibir mayores niveles de bienestar(23).

El estudio da cuenta que los estudiantes universitarios ante los diversos estresores psicosociales de la COVID-19, posiblemente tomaron una actitud proactiva que les permitió en el despliegue de sus recursos personales y anticiparse a problemas asociados a las consecuencias generadas por la pandemia(23).

Dentro de las dimensiones que componen al bienestar psicológico se encontró que la variable propósito en la vida y autoconcepto, fueron las que presentaron mayor prevalencia. El autoconcepto se relacionó con el bienestar psicológico y a su vez, estuvieron ligados a los propósitos en la vida (24), por cuanto los individuos con opiniones positivas sobre sí mismos se enfocaban más en las metas y objetivos que tenían(25) lo que favorece la identificación y activación de recursos personales al momento de enfrentar una crisis.

Probablemente y pese a que las condiciones educativas se vieron afectadas por el aislamiento social, los universitarios al tener claros sus propósitos en la vida, seguido de un autoconcepto positivo, lograron minimizar el impacto de los estresores, situación que facilitó el proceso de adaptación a las condiciones de la emergencia sanitaria(26).

En contraposición, las dimensiones que presentaron menor frecuencia fueron autonomía y relaciones positivas, ambas afectadas y alteradas por la COVID19 , puesto que al establecerse el aislamiento social obligatorio, como medida de salud pública para contrarrestar la propagación del virus, se restringió la interacción y la cohesión social de los universitarios, generando un retorno abrupto al hogar, donde la toma de decisiones recayó nuevamente sobre las figuras parentales; situación que influyó sobre su percepción de autonomía, condición experiencial básica en el pasaje de la niñez a la vida adulta(27).

En efecto, la pérdida progresiva de la autonomía, sumada a la percepción de aislamiento relacional, influye de manera negativa sobre el bienestar psicológico; siendo uno de los factores mayormente reportados por los universitarios como estresor psicosocial en el escenario de la pandemia(28). 
En lo que respecta a las estrategias de afrontamiento, se identificó que las más empleadas por los estudiantes fueron solución de problemas, reevaluación positiva, evitación emocional y evitación cognitiva. En relación a estas últimas se plantea que los sujetos que emplean la evitación emocional y cognitiva posiblemente dejan de pensar en las consecuencias negativas de la pandemia y anulan las emociones negativas asociadas a la misma; de este modo los universitarios, tienden a enfocarse en la solución y la reevaluación positiva de la situación(29).

Lo anterior evidencia que los universitarios han transitado de estrategias de afrontamiento evitativo a estrategias de afrontamiento activo, lo que refleja la disposición progresiva de estos, a vislumbrar la pandemia desde una perspectiva del aprendizaje; por el marcado locus de control externo que tuvo la pandemia como abordaje psicosocial(29).

Adicionalmente, en el estudio se identificó que las estrategias de afrontamiento menos empleadas fueron la búsqueda de apoyo profesional y espera. Esto puede deberse a que la pandemia claramente obstaculizó el acceso a servicios de salud mental, de modo que los estudiantes recurren a otro tipo de fuentes de ayuda a fin de afrontar los diversos estresores. En relación con la espera, es evidente que los universitarios tendieron a estrategias de afrontamiento activas por sobre las pasivas, debido a la gestión de la propia autonomía y los roles sociales que como adultos jóvenes había construido (1).

Una vez descritos el nivel de bienestar psicológico y las estrategias de afrontamiento empleadas por los estudiantes, se procedió a determinar la asociación entre bienestar psicológico y estrategias de afrontamiento, encontrando una asociación directa estadísticamente significativa entre el bienestar psicológico y las estrategias solución de problemas, búsqueda de apoyo social, religión, evitación cognitiva, reevaluación positiva y negación, por lo que se debe tener en cuenta que los universitarios presentan diferentes mecanismos para poder afrontar los estresores psicosociales asociados a la pandemia, produciendo un efecto de contención sobre las problemáticas asociadas al confinamiento, la educación virtual y la progresiva afectación del nicho social(30).

Posteriormente, al evaluar la asociación entre las dimensiones del bienestar psicológico y las estrategias de afrontamiento se encontró que, el autoconcepto tiene una asociación directa estadísticamente significativa con las estrategias solución de problemas, búsqueda de apoyo social, búsqueda de apoyo profesional, evitación cognitiva y reevaluación positiva. De acuerdo a lo propuesto el autoconcepto positivo está ligado con la integración social, lo cual facilita el despliegue de la estrategia de búsqueda de apoyo social(31), las relaciones positivas con los otros y el mantenimiento de relaciones estables, configura una importante fuente de apoyo social dentro de la pandemia(32), siendo las relaciones familiares positivas el principal factor protector, lo que le permite al individuo la resignificación de estresores sociales y la reevaluación permanente de las situaciones críticas, para poder hacer frente a la misma(33).

Por otra parte, las estrategias que se asociaron de forma inversa estadísticamente significativa con el autoconcepto fueron espera, evitación emocional, reacción agresiva y expresión de la dificultad de afrontamiento. Un sujeto puede presentar un autoconcepto negativo cuando se desarrolla en entornos donde prevalece la violencia verbal, física y psicológica, puesto que, aprende a enfrentar situaciones mediante el empleo de la violencia generando de este modo reacciones agresivas (34). De igual manera, el hallazgo da cuenta que en el contexto de la pandemia los universitarios, despliegan la evitación emocional cuando ven afectado su autoconcepto, de modo que logren salvaguardar la concepción y atribución positiva que tienen sobre sí mismos, eso es evitar la confrontación familiar, las dinámicas relacionales hostiles y precariedad económica derivadas del confinamiento; a su vez, el autoconcepto familiar ejerce un efecto positivo sobre el afrontamiento evitativo o activo; en ese sentido, puede señalarse que el papel de estrategias de afrontamiento como la evitación y la espera repercute de manera progresiva en la percepción y opinión que los universitarios construyeron sobre sí mismos en la pandemia(35).

La segunda dimensión del bienestar psicológico son las relaciones positivas, que se asociaron de manera directa con las estrategias de afrontamiento de espera, evitación emocional, expresión de la dificultad de afrontamiento y negación. En consecuencia, el distanciamiento social y el confinamiento causaron una ruptura en las relaciones y dinámicas sociales habituales, lo que condujo a 
percibir de manera creciente una afectación en las relaciones positivas. Para algunos autores, el establecer y mantener relaciones positivas con otras personas contribuye a que el sujeto tenga confianza en los demás, con quienes puede expresar la dificultad de afrontamiento, disminuir los efectos emocionales, lograr la contención del estresor y afrontar efectivamente el problema (36).

En cuanto a la autonomía, se encontraron relaciones directas estadísticamente significativas con las estrategias de afrontamiento espera, evitación emocional, reacción agresiva y expresión de la dificultad de afrontamiento. Se plantea que una persona autónoma puede afrontar las dificultades de forma independiente enfocándose más en la solución que en la emoción que trae consigo la situación, razón por la cual se presenta la relación entre autonomía y evitación cognitiva (37). Sin embargo, tras la pandemia se ha podido identificar que la pérdida de autonomía puede repercutir en que los universitarios expresen menos su dificultad de afrontamiento a causa de la falta de control frente a los estresores y un temor implícito en relación a la experiencia derivada de dichas emociones (38).

La cuarta dimensión correspondiente a dominio del entorno tuvo una relación directa estadísticamente significativa con las estrategias de afrontamiento solución de problemas, búsqueda de apoyo social y evitación cognitiva, Hewitt et al(39), plantean que un sujeto que presenta una buena relación con las personas que lo rodean tiene mayor posibilidad de percibir apoyo social a la hora de enfrentar una situación estresante; que juntamente con la evitación cognitiva facilita que los participantes tomen distancia de la situación y puedan disminuir la reacción cognitiva y emocional(38), es así como en relación a la pandemia, los universitarios no solo buscan apoyo social, sino intentan identificar la parte positiva del problema, tanto en el despliegue de capacidades y recursos personales, como en la activación de fuentes de apoyo familiar o social.

Frente a la dimensión propósitos de la vida se encontró una asociación directa con las estrategias de afrontamiento solución de problemas, búsqueda de apoyo social, religión, búsqueda de apoyo profesional, evitación cognitiva y reevaluación positiva. Se plantea que, el contar con apoyo social y profesional, el individuo dispone de los mecanismos de afrontamiento adecuados para atajar los problemas y proyectar metas en la vida, lo cual también puede darse desde la parte religiosa y espiritual (40).

En relación a la pandemia, las instituciones educativas se cerraron de forma física, sin embargo, esto no fue obstáculo para que se abrieran espacios de aprendizaje virtuales, las estrategias de afrontamiento del individuo mantienen una relación sinérgica y proactiva, en virtud de la cual las fuentes de apoyo pueden ayudar a que el individuo disponga de los mecanismos de afrontamiento adecuados para atajar los problemas, de modo que los estudiantes en el contexto de la pandemia pudieron evitar cognitivamente la situación y la reevaluaron de forma positiva, adaptándose con eficacia a la nueva realidad(29).

En cuanto a la dimensión crecimiento personal se asoció de manera directa con las estrategias de afrontamiento solución de problemas, evitación cognitiva, reevaluación positiva y negación. El crecimiento personal implica un continuo aprendizaje, por lo que en el escenario de la COVID19 los universitarios identificaron la capacidad para aprender de las situaciones adversas experimentadas a partir del reconocimiento de los propios recursos disponibles, con los cuales de manera progresiva hicieron un tránsito de la negación, a la solución efectiva de problemas, lo que llevo a recuperar el locus de control interno y percibir mayores niveles de bienestar psicológico en medio de la pandemia(41).

Esto pone de manifiesto que el bienestar subjetivo en tiempos de la COVID-19, incrementa en la medida de que los sujetos emplean estrategias centradas en la resolución de problemas y búsqueda de apoyo por parte de un tercero, quien puede orientar la conducta del individuo y buscar en conjunto la solución al conflicto. Frente a lo anterior una estrategia empleada por los participantes fue la religión, la cual permite que los sujetos por medio de la oración puedan sentir el apoyo de otro ser para poder enfrentar la situación $\operatorname{adversa}^{(40)}$.

Finalmente, en lo que confiere a la relación directa con la negación y evitación cognitiva, se plantea que los universitarios tienden a eliminar o neutralizar los pensamientos negativos asociados a la COVID-19 y se centran en los pensamientos positivos. De este modo, surge la reevaluación positiva del fenómeno en la que el sujeto busca aprender de la situación adversa, identificando aquellos elementos y aprendizajes 
positivos que dejó la pandemia, los cuales facilitan el afrontamiento efectivo de los estresores asociados(41).

\section{Conclusiones}

A causa de la pandemia generada por la COVID-19, los estudiantes universitarios han sufrido cambios en las dinámicas y rutinas establecidas, lo cual trajo consigo importantes impactos en su salud y bienestar psicológico. El estudio permitió vislumbrar cómo las estrategias de afrontamiento contribuyen al bienestar psicológico especialmente aquellas centradas en la solución de problemas, búsqueda de apoyo social, religión, evitación cognitiva, reevaluación positiva y negación.

Por lo anterior, se propone que en futuras investigaciones se elaboren programas de intervención para fortalecer las estrategias de afrontamiento lo cual podría generar insumos importantes para la mitigación de las consecuencias psicosociales generadas por la pandemia.

Conflicto de intereses: Ninguno declarado por los autores.

\section{Referencias}

1. Balluerka Lasa N, Gómez Benito J, Hidalgo Montesinos D, Gorostiaga Manterola A, Espada Sánchez JP, Padilla García JL, Santed Germán MÁ. Las consecuencias psicológicas de la COVID-19 y el confinamiento. Informe de investigación. [Internet]. 2020 [citado 18 julio 2020]. Disponible en: https://addi.ehu.eus/bitstream/handle/10810/45924/Con secuencias $\% 20$ psicol\%C3\%B3gicas\%20COVID-

19\%20PR3\%20DIG.pdf?sequence=1\&isAllowed=y

2. Ruiz-Roso MB, de Carvalho Padilha P, Mantilla-Escalante DC, Ulloa N, Brun P, Acevedo-Correa D, et al. Covid-19 Confinement and Changes of Adolescent's Dietary Trends in Italy, Spain, Chile, Colombia and Brazil. Nutrients [Internet]. 2020 [citado 15 Agosto 2020];12(6):1807. Disponible en: https://www.mdpi.com/2072-6643/12/6/1807

3. Pinazo-Hernandis S. Impacto psicosocial de la COVID-19 en las personas mayores: problemas y retos. Revista Española de Geriatría y Gerontología [Internet]. 2020 [citado 06 Julio 2020];55(5):249-52. Disponible en: https://www.sciencedirect.com/science/article/abs/pii/S0 211139X20300664

4. Torales J, O'Higgins M, Castaldelli J, Ventriglio A. The outbreak of COVID-19 coronavirus and its impact on global mental health. International Journal of Social Psychiatry [Internet]. 2020 [citado 18 Julio 2020];66(4):317-20. Disponible

en: https://journals.sagepub.com/doi/full/10.1177/00207640 20915212

5. Shigemura J, Ursano RJ, Morganstein JC, Kurosawa M, Benedek DM. Public responses to the novel 2019 coronavirus (2019-nCoV) in Japan: Mental health consequences and target populations. Psychiatry and Clinical Neurosciences
[Internet]. 2020 [citado 17 Agosto 2020]:74(4):281-2. DOI: 10.1111/pcn.12988.

6. Li S, Wang Y, Xue J, Zhao N, Zhu T. The impact of covid-19 epidemic declaration on psychological consequences: A study on active weibo users. International Journal of Environmental Research and Public Health [Internet]. 2020 [citado 22 septiembre 2020]:17(6):2032. Disponible en: https://www.mdpi.com/1660-4601/17/6/2032

7. Parra V, Pérez-Salas CP, Olivares H, Sáez F. Tiempos de crisis: Reposicionando la importancia de los vínculos afectivos en la escuela. Ciper [Internet]. 2020 [citado 17 julio 2020]. Disponible en: https://www.ciperchile.cl/2020/04/30/tiempos-de-crisisreposicionando-la-importancia-de-los-vinculos-afectivosen-la-escuela/

8. Duan L, Zhu G. Psychological interventions for people affected by the covid-19 epidemic. The Lancet Psychiatry [Internet]. 2020. [citado 06 agosto 2020];7(4):300-2. Disponible en: https://www.thelancet.com/journals/lanpsy/article/PIIS2 215-0366(20)30073-0/fulltext

9. Lozano-Díaz A, Fernández-Prados J, Figueredo Canosa V, Martínez-Martínez AM. Impactos del confinamiento por el COVID-19 entre universitarios: Satisfacción Vital, Resiliencia y Capital Social. RISE [Internet]. 2020. [citado 26 julio 2020];(1):79-104. Disponible en: https://hipatiapress.com/hpjournals/index.php/rise/articl e/view/5925/3119

10. Garcia-Rey T. Impacto del COVID-19 en la educación. Acta otorrinolaringología cabeza cuello [Internet]. 2020 [citado 18 agosto 2020];48(2):131-2. Disponible en: http://52.32.189.226/index.php/acorl/article/view/544/4 49

11. Pedró F. COVID-19 y educación superior en América Latina y el Caribe: efectos, impactos y recomendaciones políticas. Análisis Carolina [Internet]. 2020 [citado 20 julio 2020];36(1):1-15. Disponible en: https://fundacioncarolina.es/wpcontent/uploads/2020/06/AC-36.-2020.pdf

12. Aguilar Villanueva LF. Gobernanza y gestión pública. México: Fondo de cultura económica. 2015. Disponible en: https://marcelagonzalezduarte.files.wordpress.com/2018/ 01/aguilar-villanueva-gobernanza-y-gestion-publica.pdf

13. Sandoval Barrientos S, Dorner París A, Véliz Burgos A. Bienestar psicológico en estudiantes de carreras de la salud. Investigación en educación médica [Internet]. 2017 [citado 10 julio 2020];6(24):260-6. Disponible en: http://www.scielo.org.mx/scielo.php?script=sci_arttext\&pi $\mathrm{d}=\mathrm{S} 2007-50572017000400260$

14. Ruiz A, Díaz Arcaño K, Zaldívar Pérez D. La psicología como ciencia y profesión en el afrontamiento del COVID-19. Revista Caribeña de Psicología [Internet]. 2020 [citado 16 septiembre 2020];4(2):153-65. Disponible en: https://revistacaribenadepsicologia.com/index.php/rcp/ar ticle/view/4815

15. Lazarus R, Folkman S. Stress, Appraisal, and Coping. Nueva York: Springer; 1984.

16. Ramírez-Ortiz J, Castro-Quintero D, Lerma-Córdoba C, YelaCeballos F, Escobar-Córdoba F. Mental health consequences of the COVID-19 pandemic associated with social isolation. Colombian Journal of Anesthesiology [Internet]. 2020 [citado 18 septiembre 2020];48(4):e930. Disponible en: https://www.revcolanest.com.co/index.php/rca/article/vie $\mathrm{w} / 930$ 
17. Hernández Sampieri R, Fernández Collado C, Baptista Lucio L. Metodología de la investigación. 6 ed. México, DF: McGrawHill; 2014. Disponible en: http://observatorio.epacartagena.gov.co/wpcontent/uploads/2017/08/metodologia-de-lainvestigacion-sexta-edicion.compressed.pdf

18. Pineda Roa CA, Castro Muñoz JA, Chaparro Clavijo RA. Estudio psicométrico de las Escalas de Bienestar Psicológico de Ryff en adultos jóvenes colombianos. Pensamiento Psicológico [Internet]. 2017 [citado 20 julio 2020];16(1):4555. Disponible

en: https://revistas.javerianacali.edu.co/index.php/pensamient opsicologico/article/view/1609/2298

19. Londoño NH, Henao López GC, Puerta IC, Posada S, Arango D, Aguirre-Acevedo DC. Propiedades psicométricas y validación de la escala de estrategias de coping modificada (EEC-M) en una muestra colombiana [Internet]. Universitas Psychologica. 2006 [citado 20 julio 2020];5(2):327-50. Disponible

en: http://www.scielo.org.co/scielo.php?script=sci_arttext\&pid $=$ S1657-92672006000200010

20. Saldaña J. Ethnotheatre: Research from page to stage. New York: Routledge, 2016.

21. Roy-García I, Rivas-Ruiz R, Pérez-Rodríguez M, Palacios-Cruz L. Correlation: not all correlation entails causality. Rev Alerg Mex [Internet]. 2019 [citado 13 julio 2020];66(3):354-60. DOI: $10.29262 /$ ram.v66i3.651.

22. Colombia. Ministerio de la Protección Social. Ley 1090 de 2006, Por la cual se reglamenta el ejercicio de la profesión de la psicología, se dicta el código deontológico y bioético y otras disposiciones. Bogotá DC: Ministerio; 2006 [citado 22 mayo 2021]. Disponible

en: https://www.funcionpublica.gov.co/eva/gestornormativo/ norma.php?i=66205

23. Barrantes-Brais K, Ureña-Bonilla P. Bienestar psicológico y bienestar subjetivo en estudiantes universitarios costarricenses. Revista intercontinental de psicología y educación [Internet]. 2015 [citado 18 agosto 2020];17(1):101-23. Disponible en: https://www.redalyc.org/pdf/802/80242935006.pdf

24. Ordóñez León P. Autoconcepto Físico y Bienestar Psicológico en estudiantes de la Facultad de Psicología de la Universidad Nacional Mayor de San Marcos. Ágora revista científica. [internet]. 2015 [citado 15 julio 2020];2(2):188-95. DOI: 10.21679/arc.v2i2.38.

25. Muratori M, Zubieta E, Ubillos S, González JL, Bobowik M. Felicidad y bienestar psicológico: estudio comparativo entre Argentina y España. Psykhe [Internet]. 2015 [citado 15 julio 2020];24(2):1-18. DOI: 10.7764/psykhe.24.2.900.

26. Estrada M. Motivación, autoconcepto personal y bienestar psicológico en Cadetes de la Escuela Militar de Chorrillos. [Tesis de Doctorado]. Universidad Nacional de Educación Enrique Guzmán y Valle; 2016 [citado 10 julio 2020]. Disponible http://repositorio.une.edu.pe/handle/UNE/923

27. Nieto Álvarez J. Los cambios de la medida de 'pico y cédula' en principales ciudades [Internet]. El Tiempo. 2020 [citado 10 agosto 2020]. Disponible en: https://www.eltiempo.com/colombia/otras-ciudades/pico$\mathrm{y}$-cedula-en-colombia-novedades-en-principales-ciudades494168

28. Alania Contreras R, Llancari Morales RA, de La Cruz M, Ortega Révolo D. Adaptación del inventario cuestionario de estrés académico SISCO SV al contexto de la crisis por COVID-
19. Socialium [Internet]. 2020 [citado 10 septiembre 2020];4(2):111-30. DOI: 10.26490/uncp.sl.2020.4.2.669.

29. Luna F, Marino J, García R, Jaldo R, Leaniz AF, Foa Torres G, et al. Conectividad cerebral en la regulación emocional: La solución de problemas emocionales. Psicológica [Internet]. 2016 [citado 10 julio 2020];37(1):35-59. Disponible en: https://www.redalyc.org/pdf/169/16943586003.pdf

30. Chaves Castaño L, Orozco Rosado AM. Estilos de personalidad y estrategias de afrontamiento al estrés en soldados: un estudio correlacional. Revista de Psicología Universidad de Antioquia [Internet]. 2015 [citado 17 julio 2020];7(1):35-56. Disponible en: https://dialnet.unirioja.es/servlet/articulo? codigo $=528032$ 8

31. García Guillamón DJ, Méndez I. Autoconcepto y conductas antisociales en alumnos de Secundaria. En: Asunivep, editor. Investigación en el ámbito escolar: un acercamiento multidimensional a las variables psicológicas y educativas. 2da edición. España: Asunivep; 2016 [citado 12 julio 2020]. p. 35-40. Disponible en: https://www.eesnsrmadrededios.edu.pe/wpcontent/uploads/2020/10/Investigacion-en-el-ambitoescolar.pdf\#page $=36$

32. Blanco Abarca A, Rodríguez Marín J. Intervención Psicosocial. España: Pearson Educación; 2007.

33. García HD, Fantin MB, Pardo AY. Estrategias de afrontamiento al estrés en aspirantes a Policía de la ciudad de San Luis. Revista de Psicología: (Universidad de Antioquia) [Internet]. 2016 [citado 17 septiembre 2020];8(1):23-34. Disponible en: https://revistas.udea.edu.co/index.php/psicologia/article/ view/326890

34. Cava M, Buelga S, Carrascosa L. Violencia física y psicológica ejercida en parejas adolescentes: relación con el autoconcepto y la violencia entre iguales. Behavioral Psychology [Internet]. 2015 [citado 14 agosto 2020];23(3):429-46. Disponible en: https://go.gale.com/ps/i.do?id=GALE\%7CA521459978\&sid =googleScholar \&v=2.1\&it=r\&linkaccess=abs\&issn=113294 83\&p=AONE\&sw=w\&userGroupName=anon\%7Ee88de9be

35. Morales Rodríguez FM. Relaciones entre afrontamiento del estrés cotidiano, autoconcepto, habilidades sociales e inteligencia emocional. European Journal of Education and Psychology [Internet]. 2017 [citado 17 septiembre 2020];10(2):41-8. DOI: 10.1016/j.ejeps.2017.04.001.

36. Freire Rodríguez C. El bienestar psicológico en los estudiantes universitarios: operativización del constructo y análisis de su relación con las estrategias de afrontamiento [Tesis de Doctorado]. Universidad de A Coruña; 2014 [citado 9 septiembre 2020]. Disponible en: https://core.ac.uk/download/pdf/61913475.pdf

37. García-Alandete J. Psicología positiva, bienestar y calidad de vida. En-claves del pensamiento [internet]. 2014 [citado 6 agosto 2020];8(16):13-29. Disponible en: http://www.scielo.org.mx/scielo.php?pid=S1870879X2014000200013\&script=sci_arttext

38. Gantiva Díaz CA, Luna A, Dávila A, Salgado MJ. Estrategias de afrontamiento en personas con ansiedad. Psychologia: avances de la disciplina [Internet]. 2010 [citado 11 julio 2020];4(1):63-70. Disponible en: https://dialnet.unirioja.es/servlet/articulo? codigo $=492398$ 5

39. Hewitt Ramírez N, Juárez F, Parada Baños AJ, Guerrero Luzardo J, Romero Chávez YM, Salgado Castilla AM, et al. 
Afectaciones psicológicas, estrategias de afrontamiento y niveles de resiliencia de adultos expuestos al conflicto armado en Colombia. Revista Colombiana de Psicología [Internet]. 2016 [citado 15 julio 2020];25(1):125-40. Disponible en: https://dialnet.unirioja.es/servlet/articulo?codigo=540337 1

40. Yoffe L. Efectos positivos de las prácticas religiosas / espirituales en el duelo. Avances en Psicología [Internet].
2012 [citado 11 julio 2020];20(1):9-30. DOI: 10.33539/avpsicol.2012.v20n1.1940.

41. Marin Semanez HS, Placencia Medina MD. Motivación y satisfacción laboral del personal de una organización de salud del sector privado. Horizonte Médico [internet]. 2017 [citado 13 agosto 2020];17(4):42-52. Disponible en: http://www.scielo.org.pe/scielo.php?pid=s1727-

$558 \times 2017000400008 \&$ script=sci_arttext 